\title{
PARTITIONS WITH A RESTRICTION ON THE MULTIPLICITY OF THE SUMMANDS
}

\author{
BY \\ PETER HAGIS, JR.
}

\begin{abstract}
Using the circle dissection method, a convergent series and several asymptotic formulae are obtained for $p(n, t)$, the number of partitions of the positive integer $n$ in which no part may be repeated more than $t$ times.
\end{abstract}

1. Introduction. If $n$ and $t$ are positive integers we shall denote by $p(n, t)$ the number of partitions of $n$ in which no summand appears more than $t$ times. In particular $p(n, 1)$ is the number of partitions of $n$ into unequal parts. Several authors have already studied $p(n, 1)$ (see [3], [6], [7]), and a convergent series and asymptotic formulae for this partition function are well known. In the present paper our objective is to generalize these results and obtain a convergent series representation and asymptotic formulae for $p(n, t)$ subject only to the restriction that $n \geqq t$. Our attack is based on the familiar circle dissection method of Hardy-RamanujanRademacher.

2. The transformation equation. Since the time of Euler it has been known that the generating function of $p(n)$, the number of partitions of the positive integer $n$, is

$$
F(x)=\prod_{m=1}^{\infty}\left(1-x^{m}\right)^{-1}=\sum_{n=0}^{\infty} p(n) x^{n} .
$$

The reciprocal of $F(x)$ is

$$
H(x)=\prod_{m=1}^{\infty}\left(1-x^{m}\right)=\sum_{n=0}^{\infty} P(n) x^{n}
$$

where $P(n)$ represents the number of partitions of $n$ into an even number of distinct parts minus the number of partitions of $n$ into an odd number of distinct parts. We note (see Theorem 10.4 in [9]) that $P(n)=(-1)^{j}$ if $n=\left(3 j^{2} \pm j\right) / 2$ for some $j=0,1,2, \ldots$ and $P(n)=0$ otherwise.

The generating function of $p(n, t)$ is easily seen to be

$$
G(x, t)=\prod_{m=1}^{\infty}\left(1+x^{m}+x^{2 m}+\cdots+x^{t m}\right)=\frac{F(x)}{F\left(x^{t+1}\right)}=\sum_{n=0}^{\infty} p(n, t) x^{n} .
$$

Received by the editors May 30, 1970.

AMS 1969 subject classifications. Primary 1048.

Key words and phrases. Partitions, restrictions on multiplicity of parts, transformation equation, convergent series representation, asymptotic formulae.

Copyright (C) 1971, American Mathematical Society 
For each of the three functions just mentioned we have convergence in the interior of the unit circle.

If $h$ and $k$ are relatively prime integers with $k>0$ and $z$ is a complex number with positive real part then it is known (see [5] or [10]) that

$$
\begin{aligned}
F(\exp \{2 \pi i h / k-2 \pi z / k\}) \\
\quad=z^{1 / 2} \omega(h, k) \exp \{\pi(1 / z-z) / 12 k\} \cdot F\left(\exp \left\{2 \pi i h^{\prime} / k-2 \pi / k z\right\}\right) .
\end{aligned}
$$

$h h^{\prime} \equiv-1(\bmod k)$ and $\omega(h, k)=\exp \{\pi i s(h, k)\}$ where $s(h, k)$ is a Dedekind sum defined by $s(h, k)=\sum_{u=1}^{k}((u / k))((h u / k)) .((v))=0$ if $v$ is an integer and $((v))$ $=v-[v]-\frac{1}{2}$ otherwise.

With the aid of (2.3) we shall now derive a similar transformation equation for $G(x, t)$. In what follows $D=(k, t+1), k=D K, t+1=D T$ where, of course, $(K, T)=1$. If we take

$$
x=\exp \{2 \pi i h / k-2 \pi z / k\}
$$

then $x^{t+1}=\exp \{2 \pi i T h / K-2 \pi T z / K\}$.

Since $G(x, t)=F(x) / F\left(x^{t+1}\right)$ it follows from (2.3) that

$$
\begin{aligned}
G(x, t)= & T^{-1 / 2} w(h, k, t) \exp \{\pi(t z+(T-D) / T z) / 12 k\} \\
& \cdot F\left(\exp \left\{2 \pi i h^{\prime} / k-2 \pi / k z\right\}\right) H\left(\exp \left\{2 \pi i h^{*} / K-2 \pi / T K z\right\}\right) .
\end{aligned}
$$

Here $T h h^{*} \equiv-1(\bmod K)$, and

$$
w(h, k, t)=\omega(h, k) / \omega(T h, K) .
$$

If $T T^{\prime} \equiv 1(\bmod K)$ where $T^{\prime}$ is kept fixed and we let

$$
y=\exp \left\{2 \pi i T^{\prime} h^{\prime} / k-2 \pi / T z k\right\},
$$

then we verify without difficulty that $y^{D}=\exp \left\{2 \pi i h^{*} / K-2 \pi / T z K\right\}$, and $\exp \left\{2 \pi i h^{\prime} / k-2 \pi / k z\right\}=y^{T} \exp \left\{2 \pi i h^{\prime}\left(1-T T^{\prime}\right) / k\right\}$. If $h^{\prime} \equiv b(\bmod D)$ and $M \equiv d$ $(\bmod D)$, where $1-T T^{\prime}=M K$, then $\exp \left\{2 \pi i h^{\prime}\left(1-T T^{\prime}\right) / k\right\}=\exp \{2 \pi i b d / D\}=e(b, d, D)$. Thus, we can write $\exp \left\{2 \pi i h^{\prime} \mid k-2 \pi / k z\right\}=e(b, d, D) y^{T}$. If we define

$$
\begin{aligned}
J(y, t) & =F\left(e(b, d, D) y^{T}\right) H\left(y^{D}\right)=\sum_{n=0}^{\infty} p(n) e^{n}(b, d, D) y^{T n} \sum_{n=0}^{\infty} P(n) y^{D n} \\
& =\cdot \sum_{n=0}^{\infty} c(n, b, d, D) y^{n}
\end{aligned}
$$

we have, finally, the following:

THEOREM 1. If $x$ and $y$ are defined by (2.4) and (2.6), respectively,

$$
G(x, t)=T^{-1 / 2} w(h, k, t) \exp \{\pi(t z+(T-D) / T z) / 12 k\} J(y, t) .
$$

3. An exponential sum. In what follows we shall require an estimate of the magnitude of a certain sum involving $w(h, k, t)$. We begin by stating a proposition concerning $\omega(h, k)$ whose proof appears in [4]. 
Proposition 1. If $k$ is odd then

$$
\omega(h, k)=(h \mid k) i^{(k-1) / 2} \exp \left\{2 \pi i q\left(h-h^{\prime}\right) / g k\right\} .
$$

If $k$ is even then

$$
\omega(h, k)=(k \mid h) i^{i(k+1) / 2} \exp \left\{2 \pi i q\left(h-h^{\prime}\right) / g k\right\} .
$$

$g=(3, k)$ or $g=8(3, k)$ according as $k$ is odd or even, $h^{\prime}$ is any solution of $h h^{\prime} \equiv-1$ $(\bmod g k)$, and $q$ is any solution of $f q \equiv 1(\bmod g k)$ where $f=24 / g$. In (3.2) $b \equiv h^{\prime}$ (mod 8$)$, and the branch of $i^{b(k+1) / 2}$ is that corresponding to the principal value of the logarithm. $(a \mid c)$ is the Jacobi symbol.

Our immediate objective is to obtain a result similar to this proposition for $w(h, k, t)$. We shall utilize some elementary properties of the Jacobi symbol (see Theorems 3.5, 3.6, 3.7 in [9]) and the fact that Proposition 1 obviously holds if $h, h^{\prime}, k, g, f, q, b$ are replaced by $T h, h^{*}, K, G, F, Q, B$, respectively. Three cases must be considered.

If $k$ is odd then, of course, $K$ is also odd. It follows from (2.5) and (3.1) that

$$
w(h, k, t)=(h \mid k)(T h \mid K) i^{(k-K) / 2} \exp \left\{2 \pi i q\left(h-h^{\prime}\right) / g k\right\} \exp \left\{-2 \pi i Q\left(T h-h^{*}\right) / G K\right\} .
$$

If $T^{\prime}$ is chosen so that $T T^{\prime} \equiv 1(\bmod G K)$ we easily verify that $h^{*} \equiv T^{\prime} h^{\prime}(\bmod G K)$. If $g=J G(J=1$ or 3$)$ then $F=J f$ and $Q \equiv A q(\bmod G K)$ where $J A \equiv 1(\bmod G K)$. Also, $(h \mid k)(T h \mid K)=(h \mid D)(h \mid K)(h \mid K)(T \mid K)=(h \mid D)(T \mid K)$. We conclude that

$$
w(h, k, t)=(h \mid D)(T \mid K) i^{(k-K) / 2} \exp \left\{2 \pi i q\left(U h+V h^{\prime}\right) / g k\right\}
$$

where

$$
U=1-J A(t+1), \quad V=J A T^{\prime} D-1 .
$$

Note that $(h \mid D)(T \mid K)$ has absolute value one and depends only on $k$ and $t$ if we impose the restriction $h \equiv a(\bmod D)$ where $(a, D)=1$.

If $k$ and $K$ are both even then from (2.5) and (3.2) we have

$$
\begin{aligned}
w(h, k, t)= & (k \mid h)(K \mid T h) i^{b(k+1) / 2} i^{-B(K+1) / 2} \\
& \cdot \exp \left\{2 \pi i q\left(h-h^{\prime}\right) / g k\right\} \exp \left\{-2 \pi i Q\left(T h-h^{*}\right) / G K\right\} .
\end{aligned}
$$

Choosing $T^{\prime}$ so that $T T^{\prime} \equiv 1(\bmod G K)$ we have $h^{*} \equiv T^{\prime} h^{\prime}(\bmod G K)$ and $B \equiv h^{*}$ $\equiv T^{\prime} b(\bmod 8)($ since $8 \mid G)$. If $g=J G(J=1$ or 3$)$ then $F=J f$ and $Q \equiv A q(\bmod G K)$ where $A$ is defined as above. Also, if $D=2^{\alpha} D^{*}$ where $\alpha \geqq 0$ and $D^{*}$ is odd, then $(k \mid h)(K \mid T h)=(K \mid T)\left(2^{\alpha} \mid h\right)\left(D^{*} \mid h\right)=(K \mid T)\left(2^{\alpha} \mid h\right)\left(h \mid D^{*}\right)(-1)^{(h-1)\left(D^{*}-1\right) / 4}$; where $\left(2^{\alpha} \mid h\right)$ $=1$ if $\alpha$ is even and $\left(2^{\alpha} \mid h\right)=(-1)^{\left(h^{2}-1\right) / 8}$ if $\alpha$ is odd. Therefore,

$$
\begin{aligned}
w(h, k, t)= & (K \mid T)\left(2^{\alpha} \mid h\right)\left(h \mid D^{*}\right)(-1)^{(h-1)\left(D^{*}-1\right) / 4} i^{b(k+1) / 2} i^{-T^{\prime} b(K+1) / 2} \\
& \cdot \exp \left\{2 \pi i q\left(U h+V h^{\prime}\right) / g k\right\}
\end{aligned}
$$

where $U$ and $V$ are given by (3.4). We note that the coefficient of 
$\exp \left\{2 \pi i q\left(U h+V h^{\prime}\right) / g k\right\}$ has absolute value one and depends only on $k$ and $t$ if we impose the restrictions $h \equiv a(\bmod D)$ where $(a, D)=1$, and $h \equiv d(\bmod 8)$ where $d$ is odd.

If $k$ is even and $K$ is odd then

$$
\begin{aligned}
w(h, k, t)= & (k \mid h)(T h \mid K) i^{b(k+1) / 2} i^{-(K-1) / 2} \\
& \cdot \exp \left\{2 \pi i q\left(h-h^{\prime}\right) / g k\right\} \exp \left\{-2 \pi i Q\left(T h-h^{*}\right) / G K\right\} .
\end{aligned}
$$

As before $h^{*} \equiv T^{\prime} h^{\prime}(\bmod G K)$. If $g=J G(J=8$ or 24$)$ then $F=J f$ and $Q \equiv A q$ $(\bmod G K)$ where $J A \equiv 1(\bmod G K)$. Writing $D=2^{\alpha} D^{*}$ we have

$$
\begin{aligned}
(k \mid h)(T h \mid K) & =(D \mid h)(K \mid h)(h \mid K)(T \mid K) \\
& =(D \mid h)(T \mid K)(-1)^{(h-1)(K-1) / 4} \\
& =(T \mid K)\left(2^{\alpha} \mid h\right)\left(h \mid D^{*}\right)(-1)^{(h-1)\left(D^{*}-1\right) / 4}(-1)^{(h-1)(K-1) / 4} \\
& =(T \mid K)\left(2^{\alpha} \mid h\right)\left(h \mid D^{*}\right)(-1)^{(h-1)\left(K-D^{*}\right) / 4} .
\end{aligned}
$$

We conclude that

$$
\begin{aligned}
w(h, k, t)= & (T \mid K)\left(2^{\alpha} \mid h\right)\left(h \mid D^{*}\right)(-1)^{(h-1)\left(K-D^{*}\right) / 4} i^{b(k+1) / 2} i^{-(K-1) / 2} \\
& \cdot \exp \left\{2 \pi i q\left(U h+V h^{\prime}\right) / g k\right\}
\end{aligned}
$$

where $U$ and $V$ are given by (3.4). The coefficient of $\exp \left\{2 \pi i q\left(U h+V h^{\prime}\right) / g k\right\}$ has absolute value one and depends only on $k$ and $t$ if $h \equiv a(\bmod D)$ where $(a, D)=1$, and $h \equiv d(\bmod 8)$ where $d$ is odd.

We summarize (3.3), (3.4), (3.5), (3.6) in the following proposition. All undefined symbols have the meanings given earlier in this section.

Proposition 2. $w(h, k, t)=C(h, k, t) \exp \left\{2 \pi i q\left(U h+V h^{\prime}\right) / g k\right\}$ where $|C(h, k, t)|$ $=1$. Furthermore, $C(h, k, t)$ depends only on $k$ and $t$ if $h \equiv a(\bmod D)$ where $(a, D)=1$ and also, if $k$ is even, $h \equiv d(\bmod 8)$ where $d$ is odd. If $g=J G(J=1,3,8,24)$ and $J A \equiv 1(\bmod G K)$, then $U=1-J A(t+1)$ and $V=J A T^{\prime} D-1$.

We are now prepared to prove the main result of this section.

THEOREM 2. If $(k, t+1)=D, h \equiv a(\bmod D),(a, D)=1 ; h h^{\prime} \equiv-1(\bmod k)$, $s_{1} \leqq h^{\prime}<s_{2}(\bmod k), 0 \leqq s_{1}<s_{2} \leqq k ; t \leqq n ;$ and $M$ is a fixed integer, then the sum

$$
Y=\sum_{h \bmod k}^{\prime} w(h, k, t) \exp \left\{-2 \pi i\left(h n-h^{\prime} M\right) / k\right\}
$$

is subject to the estimate $O\left(n^{1 / 3} k^{2 / 3}+\varepsilon\right)$ where the multiplicative constant implied by the $O$-symbol depends only on $t$. The symbol $\sum^{\prime}$ indicates that the variable of summation runs through a reduced residue system of the given modulus subject, perhaps, to some other stated restrictions.

Proof. Since $w(h, k, t)$ has period $k$ when viewed as a function of $h$, if we change 
the modulus in $Y$ to $g k$ and select $h^{\prime}$ so that $h h^{\prime} \equiv-1(\bmod g k)$, then by Proposition 2

$$
Y=g^{-1} \sum_{h \bmod g k}^{\prime} C(h, k, t) \exp \{2 \pi i f(h) / g k\}
$$

where $f(h)=(q U-g n) h+(q V+g M) h^{\prime}$.

If $k$ is even we split $Y$ into four parts, $Y_{1}, Y_{3}, Y_{5}, Y_{7}$, so that in $Y_{d}$ we have $h \equiv d(\bmod 8)($ as well as $h \equiv a(\bmod D))$. Then $Y=Y_{1}+Y_{3}+Y_{5}+Y_{7}$ where

$$
Y_{d}=C_{d} \sum_{h \bmod g k}^{\prime} \exp \{2 \pi i f(h) / g k\}
$$

with $\left|C_{d}\right|=g^{-1} \leqq 1$.

If $k$ is odd then (3.7) holds if we identify $Y$ with $Y_{d}$ and ignore the restriction $h \equiv d(\bmod 8)$.

If we define the function $m(s)$ for all integers $s$ by requiring that $m(s)=1$ if $s_{1} \leqq s<s_{2}(\bmod k)$, and $m(s)=0$ otherwise, then $m(s)$ has period $k$. From the theory of finite Fourier series we have $m(s)=\sum_{j=0}^{k-1} \alpha_{j} \exp \{2 \pi i s j \mid k\}$ where $\alpha_{j}=$ $k^{-1} \sum_{s=0}^{k-1} m(s) \exp \{-2 \pi i s j / k\}$. It is not difficult to prove (see $\$ 10$ in [8]) that $\sum_{j=0}^{k-1} \alpha_{j}=O(\log k)$ so that $\sum_{j=0}^{k-1} \alpha_{j}=O\left(k^{\varepsilon}\right)$ for any $\varepsilon>0$.

We can now drop the restriction $s_{1} \leqq h^{\prime}<s_{2}(\bmod k)$ and write

$$
\begin{aligned}
Y_{d} & =C_{d} \sum_{h \bmod g k}^{\prime} m\left(h^{\prime}\right) \exp \{2 \pi i f(h) / g k\} \\
& =C_{d} \sum_{j=0}^{k-1} \alpha_{j} \sum_{h \bmod g k}^{\prime} \exp \left\{2 \pi i\left((q U-g n) h+(q V+g M+g j) h^{\prime}\right) / g k\right\} .
\end{aligned}
$$

If $k$ is odd then $\Sigma^{\prime}$ is a Kloosterman sum. If $k$ is even we write $D=2^{\alpha} D^{*}$ where $\alpha \geqq 0$ and $D^{*}$ is odd. It is easy to see that if $\alpha \leqq 1$ then the two conditions (I) $h \equiv a$ $(\bmod D)$ and (II) $h \equiv d(\bmod 8)$ are equivalent to a single condition of the form $h \equiv a^{*}\left(\bmod 8 D^{*}\right)$. If $\alpha=2$ and $a \neq d(\bmod 4)$ then $\Sigma^{\prime}$ is empty. If $a \equiv d(\bmod 4)$ then (I) and (II) are equivalent to $h \equiv a^{*}\left(\bmod 8 D^{*}\right)$. If $\alpha \geqq 3$ and $a \neq \equiv d(\bmod 8)$ then $\Sigma^{\prime}$ is empty, while if $a \equiv d(\bmod 8)$ then (I) and (II) are equivalent to $h \equiv a(\bmod D)$. Thus, we see that in each case either $\Sigma^{\prime}$ is empty or $\Sigma^{\prime}$ is a Kloosterman sum. Using a theorem of Salié [12] it follows that

$$
|Y|<C_{0} k^{2 / 3+\varepsilon}(q U-g n, g k)^{1 / 3}
$$

where $C_{0}$ is a constant which is independent of all the parameters involved.

Since $(f, g k)=1$, we have $(q U-g n, g k)=(f q U-f g n, g k)$. But $f g=24$ and $f q U$ $=U+m g k$ where $m$ is an integer. Therefore, $(q U-g n, g k)=(U-24 n, g k)$ $\leqq D g(U-24 n, K)$. From Proposition 2 we see that $U=1-(t+1)(1+p G K)$ $=-t+P K$, so that $(q U-g n, g k) \leqq D g(t+24 n, K) \leqq D g(t+24 n) \leqq 25 D g n$. The conclusion of the theorem now follows from (3.8). 
4. A convergent series for $p(n, t)$. Applying Cauchy's integral formula to $G(x, t)$ we have

$$
2 \pi i p(n, t)=\int_{C} x^{-n-1} G(x, t) d x=\sum_{n, k}^{\prime} \int_{\xi n k} x^{-n-1} G(x, t) d x .
$$

Here $0 \leqq h<k \leqq N,(h, k)=1$, and $\xi_{h k}$ are the Farey arcs of order $N$ of $C$, the circle $|x|=\exp \left\{-2 \pi N^{-2}\right\}$. If, on the arc $\xi_{n k}$, we let $x=\exp \{2 \pi i h / k-2 \pi z / k\}$ where $z=w k, w=N^{-2}-i \theta$, we obtain

$$
\text { (4.1) } p(n, t)=\sum_{n, k}^{\prime} \exp \{-2 \pi i n h / k\} \int G(\exp \{2 \pi i h / k-2 \pi z / k\}, t) \exp \{2 \pi n w\} d \theta \text {. }
$$

The limits of integration are $-1 / k\left(k+k_{1}\right)$ and $1 / k\left(k+k_{2}\right)$ where $k_{1}, k, k_{2}$ are the denominators of consecutive terms of the Farey series of order $N$.

If $D$ runs through the positive divisors of $t+1$, and $a$ runs through a reduced residue system modulo $D$, we have

$$
p(n, t)=\sum_{D \mid t+1} \sum_{a \bmod D}^{\prime} \sum_{d=1}^{D} S(D, a, d)
$$

where $S(D, a, d)$ denotes the sum of all those terms in (4.1) which satisfy the conditions $(k, t+1)=D, h \equiv a(\bmod D)$, and $M \equiv d(\bmod D)$ where $1-T T^{\prime}=M K($ see the remarks just preceding (2.7)). Notice that if $a b \equiv-1(\bmod D)$ and $h h^{\prime} \equiv-1$ $(\bmod k)$, then $h^{\prime} \equiv b(\bmod D)$ in $S(D, a, d)$. Now either $S(D, a, d)=0$ or we have from (2.8), (2.7), (2.6)

$$
\begin{aligned}
S(D, a, d)= & T^{-1 / 2} \sum_{h, k}^{\prime} w(h, k, t) \exp \{-2 \pi i n h / k\} \\
& \cdot \int \sum_{j=0}^{\infty} c(j, b, d, D) \exp \left\{2 \pi i h^{\prime} T^{\prime} j / k\right\} \\
& \cdot \exp \left\{-\left(\pi / k^{2} w T\right)(2 j-(T-D) / 12)+\pi w(2 n+t / 12)\right\} d \theta .
\end{aligned}
$$

The limits of integration are as before, $1 \leqq k \leqq N,(k, t+1)=D, M \equiv d, h \equiv a$, $h^{\prime} \equiv b$ (all modulo $D$ ) and $1-T T^{\prime}=M K$. We note that $c(j, b, d, D)$ depends only on $j$ here.

We now split $S(D, a, d)$ into two parts, $Q(D, a, d)$ and $R(D, a, d)$, according as $j<(T-D) / 24$ or $j \geqq(T-D) / 24$, respectively. Employing Rademacher's argument [11] and making use of Theorem 2 we find that

$$
R(D, a, d)=O\left(n^{1 / 3} N^{-1 / 3+\varepsilon} \exp \left\{2 \pi n N^{-2}\right\}\right) .
$$

Here, and in the remainder of this section, the multiplicative constant implied by the $O$-notation depends at most on $t$.

In $Q(D, a, d)$ the condition that $j<(T-D) / 24$ implies that $Q(D, a, d)=0$ if $T \leqq D$. Since $D T=t+1$ this will occur if, and only if, $D \geqq(t+1)^{1 / 2}$. We therefore consider only those $D$ such that $D<(t+1)^{1 / 2}$. 
Proceeding as in [11] we obtain

$$
\begin{aligned}
Q(D, a, d)= & 2 \pi T^{-1} \sum_{k} \sum_{j} c(j, b, d, D) A\left(k, t, n, j, a, T^{\prime}\right) L^{*}(k, t, n, j) \\
& +O\left(n^{1 / 3} N^{-1 / 3+\varepsilon} \exp \left\{2 \pi n N^{-2}\right\}\right)
\end{aligned}
$$

where $1 \leqq k \leqq N, 0 \leqq j<(T-D) / 24$, and the other restrictions mentioned earlier are still in force.

$$
A\left(k, t, n, j, a, T^{\prime}\right)=\sum_{h \bmod k}^{\prime} w(h, k, t) \exp \left\{-2 \pi i\left(n h-T^{\prime} j h^{\prime}\right) / k\right\}
$$

where $h \equiv a(\bmod D)$.

$$
\begin{aligned}
L^{*}(k, t, n, j)= & k^{-1}\{(T-D-24 j) /(24 n+t)\}^{1 / 2} \\
& \cdot I_{1}\left\{\pi(24 n+t)^{1 / 2}(T-D-24 j)^{1 / 2} / 6 k T^{1 / 2}\right\}
\end{aligned}
$$

where $I_{1}(x)$ is the Bessel function of order one.

Since $D<T$ we see from (2.7) that if $j<(T-D) / 24$ then $c(j, b, d, D)=P(j / D)$ if $D \mid j$ and $c(j, b, d, D)=0$ if $D \nmid j$. Therefore, if we let $j=D m$ and write

$$
J=(T-D) / 24 D, \quad r=t / 24,
$$

we have from (4.2), (4.3), (4.4), first summing over $d, a$, and $D$, and then letting $N$ approach infinity,

THEOREM 3. The number of partitions of the positive integer $n$ in which no part appears more than $t$ times has the following infinite series representation:

$$
p(n, t)=2 \pi(t+1)^{-1} \sum_{D} \sum_{k} \sum_{m<J} P(m) A(k, t, n, m) L(k, t, n, m) .
$$

Here $D \mid(t+1)$ and $D<(t+1)^{1 / 2} ;(k, t+1)=D ; P(m)=0,1,-1$, according to the rule given at the beginning of $\$ 2$;

$$
\begin{aligned}
A(k, t, n, m)= & \sum_{n \bmod k}^{\prime} w(h, k, t) \exp \left\{-2 \pi i\left(n h-D T^{\prime} m h^{\prime}\right) / k\right\} ; \\
L(k, t, n, m)= & D^{3 / 2} k^{-1}\{(J-m) /(n+r)\}^{1 / 2} \\
& \cdot I_{1}\left\{4 \pi D k^{-1}((J-m)(n+r) /(t+1))^{1 / 2}\right\}
\end{aligned}
$$

where $J$ and $r$ are given by (4.5).

We remark that Theorem 3 can be given a different interpretation. For according to a theorem of Glaisher [1] $p(n, t)$ also represents the number of partitions of $n$ having the property that no part is divisible by $t+1$. From this point of view we also observe that Theorem 9 of [2] is the special case of Theorem 3 when $t+1$ is an odd prime.

5. Some special cases. If $t \leqq 24$ and $t=p^{j}-1$, where $p$ is a prime and $j=1$ or 2 , then in (4.6) only $D=1$ and $m=0$ appear. Also, $T=p^{j}$ and $J=r=t / 24$ so that we have 
COROLlaRY 3.1. If $t \leqq 24$ and $t=p^{j}-1, p$ a prime and $j=1$ or 2 , then

$$
p(n, t)=2 \pi p^{-j} \sum_{k} k^{-1}\{t /(t+24 n)\}^{1 / 2} A(k, t, n, 0) I_{1}\left\{\pi\left(t^{2}+24 n t\right)^{1 / 2} /\left(6 k p^{j / 2}\right)\right\}
$$

where $(p, k)=1$.

If, in particular, $t=1$, we have

COROLlARY 3.2. The number of partitions of a positive integer $n$ into unequal parts is given by

$$
p(n, 1)=\pi \sum_{k} k^{-1}(24 n+1)^{-1 / 2} A(k, 1, n, 0) I_{1}\left\{\pi(48 n+2)^{1 / 2} / 12 k\right\}
$$

where $2 \nmid k$.

This result agrees with Theorem 4 in [3]. (It is not difficult to show that $A(k, 1, n, 0)$ here and $B(k, n)$ in [3] are equal.)

6. Asymptotic formulae. In this section $c$ denotes a positive constant, and both $c$ and the multiplicative constant implied by the $O$-symbol depend at most on $t$.

If we write

(6.1) $r=t / 24$,

(6.2) $G(m)=(r-m)^{1 / 2}$,

(6.3) $E=4 \pi(n+r)^{1 / 2}$,

(6.4) $s=(t+1)^{-1 / 2}$,

(6.5) $W=\sum_{m<r} P(m) G(m) I_{1}\{s E G(m)\}$,

then from (4.6) we have, splitting off the term for which $k=1$,

$$
p(n, t)=2 \pi s^{2} W(n+r)^{-1 / 2}(1+S) \text {. }
$$

Here,

$$
\begin{aligned}
S= & \sum_{D} \sum_{k>1} \sum_{m<J} P(m) A(k, t, n, m) D^{3 / 2}(k W)^{-1}(J-m)^{1 / 2} \\
& \cdot I_{1}\left\{D E s k^{-1}(J-m)^{1 / 2}\right\}
\end{aligned}
$$

where $D \mid(t+1), D<(t+1)^{1 / 2},(k, t+1)=D$, and $J$ is given by (4.5).

We shall prove that for large $n$

$$
S=O\left(\exp \left\{-c n^{1 / 2}\right\}\right)
$$

which, in conjunction with (6.6), yields

THEOREM 4. As $n \rightarrow \infty$,

$$
p(n, t)=2 \pi s^{2} W(n+r)^{-1 / 2}\left(1+O\left(\exp \left\{-c n^{1 / 2}\right\}\right)\right)
$$

where $r, s, W$ are given by (6.1), (6.4), (6.5), respectively.

For the proof of (6.8) we require two lemmas. The first is a restatement of some well-known results from the theory of Bessel functions. We shall prove the second. 
LEMMA 1. If $x$ is real and positive then

(6.10) $I_{1}(x)$ is a positive, monotonic increasing function of $x$,

(6.11) $I_{1}(x)=O(x)$ if $x<1$,

(6.12) $I_{1}(x)=e^{x}(2 \pi x)^{-1 / 2}\left(1+O\left(x^{-1}\right)\right)$ if $x>1$.

LEMMA 2. If $W$ is given by (6.5) then for large $n$

$$
W=r^{1 / 2} I_{1}\left\{s E r^{1 / 2}\right\}\left(1+O\left(\exp \left\{-c n^{1 / 2}\right\}\right)\right) .
$$

Proof. We assume that $t>24$ since otherwise the result is immediate. From (6.5) we have

$$
W=r^{1 / 2} I_{1}\left\{s E r^{1 / 2}\right\}\left(1+\sum_{0<m<r} P(m)(1-m / r)^{1 / 2} I_{1}\{s E G(m)\} / I_{1}\left\{s E r^{1 / 2}\right\}\right) .
$$

From (6.10), (6.12), (6.2), (6.3) it follows that for large $n$

$$
\begin{aligned}
I_{1}\{s E G(m)\} / I_{1}\left\{s E r^{1 / 2}\right\} & =O\left(\exp \left\{s E\left(G(1)-r^{1 / 2}\right)\right\}\right) \\
& =O\left(\exp \left\{-4 \pi s n^{1 / 2}(1+r / n)^{1 / 2}\left(r^{1 / 2}-(r-1)^{1 / 2}\right)\right\}\right) \\
& =O\left(\exp \left\{-c n^{1 / 2}\right\}\right) .
\end{aligned}
$$

Since $\left|P(m)(1-m / r)^{1 / 2}\right|<1(6.13)$ follows from (6.14) and (6.15), and the proof of the lemma is complete.

From (4.7) and Theorem 2 we see that $A(k, t, n, m)=O\left(n^{1 / 3} k^{2 / 3+\varepsilon}\right)$. Therefore, from (6.7), (6.10), (6.13) and the fact that $|P(m)| \leqq 1$ we have for large $n$

$$
S=O\left(\sum_{k=2}^{\infty} n^{1 / 3} k^{-1 / 3+8} I_{1}\left\{D E s J^{1 / 2} k^{-1}\right\} / I_{1}\left\{s E r^{1 / 2}\right\}\right)
$$

But $\quad D s J^{1 / 2}=D(t+1)^{-1 / 2}((T-D) / 24 D)^{1 / 2}=T^{-1 / 2}((T-D) / 24)^{1 / 2}<(24)^{-1 / 2}=\beta$, so that

$$
S=O\left(\sum_{k=2}^{\infty} n^{1 / 3} k^{-1 / 3+\varepsilon} I_{1}\{E \beta / k\} / I_{1}\left\{s E r^{1 / 2}\right\}\right) .
$$

Splitting the sum over $k$ into two parts according as $k \leqq[E \beta]=X$ or $k>X$ and using Lemma 1 we have

$$
\begin{aligned}
S= & O\left(\sum_{k=2}^{X} n^{1 / 3} k^{1 / 6+\varepsilon} \exp \left\{-E\left(s r^{1 / 2}-\beta / k\right)\right\}\right) \\
& +O\left(\sum_{k>X} n^{13 / 12} k^{-4 / 3+\varepsilon} \exp \left\{-E s r^{1 / 2}\right\}\right)
\end{aligned}
$$

Since $s r^{1 / 2}=\beta(t /(t+1))^{1 / 2} \geqq \beta / 2^{1 / 2}$ and $-\beta / k \geqq-\beta / 2$ we see that $-E\left(s r^{1 / 2}-\beta / k\right)$ $<-c n^{1 / 2}$ for large $n$. Also, $-E s r^{1 / 2}<-c n^{1 / 2}$. We now obtain easily

$$
S=O\left(n^{11 / 12+8} \exp \left\{-c n^{1 / 2}\right\}\right),
$$

and (6.8) follows from the observation that $n=O\left(\exp \left\{.5 c n^{1 / 2}\right\}\right)$.

From Theorem 4 and Lemma 2 we have 
Corollary 4.1. As $n \rightarrow \infty$

$$
p(n, t)=2 \pi s^{2} r^{1 / 2}(n+r)^{-1 / 2} I_{1}\left\{s E r^{1 / 2}\right\}\left(1+O\left(\exp \left\{-c n^{1 / 2}\right\}\right)\right) .
$$

Finally, from Corollary 4.1 and (6.12) we obtain

Corollary 4.2. As $n \rightarrow \infty$

$$
p(n, t)=12^{1 / 2} s^{3 / 2} t^{1 / 4}(24 n+t)^{-3 / 4} \exp \left\{s E r^{1 / 2}\right\}\left(1+O\left(n^{-1 / 2}\right)\right) .
$$

\section{REFERENCES}

1. J. W. L. Glaisher, $A$ theorem in partitions, Messenger Math. 12 (1883), 158-170.

2. P. Hagis, Jr., A problem on partitions with a prime modulus $p \geqq 3$, Trans. Amer. Math. Soc. 102 (1962), 30-62. MR $26 \# 3688$.

3. —_ Partitions into odd summands, Amer. J. Math. 85 (1963), 213-222. MR 27 \#3613.

4. - - A root of unity occurring in partition theory, Proc. Amer. Math. Soc. 26 (1970), 579-582.

5. G. H. Hardy and S. Ramanujan, Asymptotic formulae in combinatorial analysis, Proc. London Math. Soc. (2) 17 (1918), 75-115.

6. L. K. Hua, On the number of partitions of a number into unequal parts, Trans. Amer. Math. Soc. 51 (1942), 194-201. MR 3, 270.

7. S. Iseki, A partition function with some congruence condition, Amer. J. Math. 81 (1959), 939-961. MR 21 \#7189.

8. J. Lehner, A partition function connected with the modulus five, Duke Math. J. 8 (1941), 631-655. MR 3, 166.

9. I. Niven and H. S. Zuckerman, An introduction to the theory of numbers, 2nd ed., Wiley, New York, 1966. MR 33 \#3981.

10. H. Rademacher, Zur Theorie der Modulfunktionen, J. Reine Angew. Math. 167 (1931), 312-336.

11. _ The Fourier coefficients of the modular invariant $J(\tau)$, Amer. J. Math. 60 (1938), 501-512.

12. H. Salié, Zur Abschätzung der Fourierkoeffizienten ganzer Modulformen, Math. Z. 36 (1933), 263-278.

TEMPLE UNIVERSITY,

Philadelphia, Pennsylvania 19122 